\title{
THE SOLAR NEIGHBORHOOD. XXX. FOMALHAUT C
}

\author{
Eric E. MamajeK ${ }^{1,2}$, Jennifer L. Bartlett ${ }^{3}$, Andreas Seifahrt $^{4}$, Todd J. Henry ${ }^{5}$, Sergio B. Dieterich $^{5}$, \\ John C. Lurie ${ }^{5}$, Matthew A. Kenworthy ${ }^{6}$, Wei-Chun JaO ${ }^{5}$, Adric R. Riedel ${ }^{7,8}$, John P. Subasavage ${ }^{9}$, \\ Jennifer G. Winters ${ }^{5}$, Charlie T. FinCh ${ }^{3}$, Philip A. IANna ${ }^{10}$, And JaCOB Bean ${ }^{4}$ \\ ${ }^{1}$ Department of Physics and Astronomy, University of Rochester, Rochester, NY 14627, USA; emamajek@ pas.rochester.edu \\ ${ }^{2}$ Cerro Tololo Inter-American Observatory, Casilla 603, La Serena, Chile \\ ${ }^{3}$ US Naval Observatory, 3450 Massachusetts Avenue, NW, Washington, DC 20392, USA \\ ${ }^{4}$ Department of Astronomy and Astrophysics, University of Chicago, Chicago, IL 60637, USA \\ ${ }^{5}$ Department of Physics and Astronomy, Georgia State University, Atlanta, GA 30302-4106, USA \\ ${ }^{6}$ Leiden Observatory, Leiden University, P.O. Box 9513, 2300-RA Leiden, The Netherlands \\ ${ }^{7}$ Department of Physics and Astronomy, Hunter College, 695 Park Avenue, New York, NY 10065, USA \\ ${ }^{8}$ Department of Astrophysics, American Museum of Natural History, Central Park West at 79th Street, New York, NY 10034, USA \\ ${ }^{9}$ US Naval Observatory, Flagstaff Station, P.O. Box 1149, Flagstaff, AZ 86002-1149, USA \\ ${ }^{10}$ Department of Astronomy, University of Virginia, P.O. Box 400325, Charlottesville, VA 22904-4325, USA \\ Received 2013 April 14; accepted 2013 September 30; published 2013 November 8
}

\begin{abstract}
LP 876-10 is a nearby active M4 dwarf in Aquarius at a distance of 7.6 pc. The star is a new addition to the 10 pc census, with a parallax measured via the REsearch Consortium On Nearby Stars (RECONS) astrometric survey on the Small and Moderate Aperture Research Telescope System's $0.9 \mathrm{~m}$ telescope. We demonstrate that the astrometry, radial velocity, and photometric data for LP 876-10 are consistent with the star being a third bound stellar component to the Fomalhaut multiple system, despite the star lying nearly $6^{\circ}$ away from Fomalhaut A in the sky. The three-dimensional separation of LP 876-10 from Fomalhaut is only $0.77 \pm 0.01 \mathrm{pc}$, and $0.987 \pm 0.006 \mathrm{pc}$ from TW PsA (Fomalhaut B), well within the estimated tidal radius of the Fomalhaut system (1.9 pc). LP 87610 shares the motion of Fomalhaut within $\sim 1 \mathrm{~km} \mathrm{~s}^{-1}$, and we estimate an interloper probability of $\sim 10^{-5}$. Neither our echelle spectroscopy nor astrometry are able to confirm the close companion to LP 876-10 reported in the Washington Double Star Catalog (WSI 138). We argue that the Castor Moving Group to which the Fomalhaut system purportedly belongs, is likely to be a dynamical stream, and hence membership to the group does not provide useful age constraints for group members. LP 876-10 (Fomalhaut C) has now risen from obscurity to become a rare example of a field $\mathrm{M}$ dwarf with well-constrained age (440 $\pm 40 \mathrm{Myr})$ and metallicity. Besides harboring a debris disk system and candidate planet, Fomalhaut now has two of the widest known stellar companions.
\end{abstract}

Key words: binaries: visual - stars: activity - stars: fundamental parameters - stars: individual (LP 876-10, Fomalhaut, TW PsA) - stars: rotation

Online-only material: color figure

\section{INTRODUCTION}

Fomalhaut is an important nearby A3 V star, containing a large resolved dusty debris disk (Gillett 1986; Kalas et al. 2005) and a candidate extrasolar planet (Kalas et al. 2008, 2013; Quillen 2006; Currie et al. 2012). Fomalhaut has previously had at least two stars suggested to be companions. See (1898) reported a 14th magnitude stellar companion to Fomalhaut at $30^{\prime \prime}$ separation; however, this star was later deemed a background star by Burnham (1978). ${ }^{11}$ Luyten (1938) reported discovery of a K-type common proper motion companion to Fomalhaut: TW PsA (HR 8721). The physicality of the Fomalhaut-TW PsA binary system was investigated by Barrado y Navascues et al. (1997) and Mamajek (2012), and both studies concluded that the pair comprise a physical binary.

\footnotetext{
11 See (1898) reported a single observation of a 14th magnitude companion at $\theta=36.2$, separation 29 ".98, and epoch 1896.706. Dubbed " $\lambda_{1} 478$ " by See, this object appears to have largely disappeared from the literature, and does not appear in the modern Washington Double Star catalog. The only subsequent mentions that we found of this companion are in the Burnham (1906) compendium of double stars (Entry 12071 is listed as "See 478"), and in two popular books (Allen 1963; Burnham 1978). Burnham (1978) stated "it appears to be merely a faint field star, having no real connection with Fomalhaut." Based on the van Leeuwen (2007) Hipparcos astrometry for Fomalhaut A, we estimate that Fomalhaut A has moved 35" since See's observation, and was at International Celestial Reference System (ICRS)
}

Mamajek (2012) estimated that Fomalhaut and TW PsA have a true separation of only $0.28 \mathrm{pc}$ and share velocities within $0.1 \pm 0.5 \mathrm{~km} \mathrm{~s}^{-1}$, consistent with constituting a bound system. Mamajek (2012) estimated the age of the Fomalhaut binary system to be $440 \pm 40$ Myr based on multiple age indicators, with the isochronal age of Fomalhaut A and the gyrochronology age of Fomalhaut B providing the most weight.

During the preparation of the Mamajek (2012) article, another neighboring star was identified that appeared to share motion with Fomalhaut and TW PsA: LP 876-10 (NLTT 54872, WT 2282, 2MASS J22480446-2422075, and PM I22480-2422). LP $876-10$ is a high proper motion star first cataloged as such by Luyten \& Hughes (1980), situated 5.67 NW (20407".6; P.A. $=337.91)$ of Fomalhaut. At the time of writing Mamajek (2012) there was insufficient evidence to test whether LP 876-10

position 22:57:36.44-29:37:03.0 at epoch 1896.706. See's reported position angle and offset corresponds to $\Delta \alpha=+17^{\prime \prime} .7, \Delta \delta=+24^{\prime \prime} .2$, hence if this object were stationary, we would predict its ICRS position to be near 22:57:37.829:36:39. No cataloged object appears near this position. Examination of Figure 3 of Marengo et al. (2009), an IRAC $4.5 \mu \mathrm{m}$ full-array, roll-subtracted image taken with Spitzer Space Telescope, shows no obvious point source either at the position See reported, nor where See's star would appear if it were comoving with Fomalhaut. Given that (1) See only reported a single observation, (2) no subsequent literature characterized the object, and (3) we were unable to find the star in the Spitzer imagery and other modern catalogs, we conclude that See's reported companion to Fomalhaut was likely spurious. 
Table 1

Stellar Parameters

\begin{tabular}{|c|c|c|c|c|c|}
\hline (1) & (2) & (3) & (4) & (5) & (6) \\
\hline Value & $\alpha$ PsA & TW PsA & LP 876-10 & Units & Ref. \\
\hline$\ldots$ & Fomalhaut A & Fomalhaut B & Fomalhaut C & $\cdots$ & \\
\hline$\alpha_{\mathrm{ICRS}}(\mathrm{J} 2000)$ & 344.411773 & 344.099277 & 342.018632 & deg & $1,1,2$ \\
\hline$\delta_{\text {ICRS }}(\mathrm{J} 2000)$ & -29.621837 & -31.565179 & -24.368872 & deg & $1,1,2$ \\
\hline Parallax & $129.81 \pm 0.47$ & $131.42 \pm 0.62$ & $132.07 \pm 1.19$ & mas & $1,1,3$ \\
\hline Distance & $7.704 \pm 0.028$ & $7.609 \pm 0.036$ & $7.572 \pm 0.068$ & $\mathrm{pc}$ & $1,1,3$ \\
\hline$\mu_{\alpha}$ & $329.95 \pm 0.50$ & $331.11 \pm 0.65$ & $333.8 \pm 0.5$ & mas $\mathrm{yr}^{-1}$ & $1,1,3$ \\
\hline$\mu_{\delta}$ & $-164.67 \pm 0.35$ & $-158.98 \pm 0.48$ & $-177.5 \pm 0.7$ & $\operatorname{mas} \mathrm{yr}^{-1}$ & $1,1,3$ \\
\hline$v_{R}$ & $6.5 \pm 0.5$ & $6.6 \pm 0.1$ & $6.5 \pm 0.5$ & $\mathrm{~km} \mathrm{~s}^{-1}$ & $4,5,3$ \\
\hline$m_{V}$ & $1.155 \pm 0.005$ & $6.488 \pm 0.012$ & $12.62 \pm 0.01$ & mag & $6,6,3$ \\
\hline$M_{V}$ & $1.72 \pm 0.01$ & $7.08 \pm 0.02$ & $13.21 \pm 0.02$ & mag & $3,3,3$ \\
\hline Period $_{\text {Rot }}$ & $\ldots$ & 10.3 & 0.466 & day & 7,3 \\
\hline SpT & A3 Va & $\mathrm{K} 4 \mathrm{e}$ & M4 V & $\ldots$ & $8,9,10$ \\
\hline$T_{\text {eff }}$ & $8590 \pm 73$ & $4594 \pm 80$ & $3132 \pm 65 \mathrm{~K}$ & $\mathrm{~K}$ & $11,12,3$ \\
\hline$f_{\text {bol }}$ & $8.96 \pm 0.25$ & 0.10075 & $0.00257(5)$ & $\mathrm{nW} \mathrm{m}^{-2}$ & $13,12,3$ \\
\hline$X_{\text {gal }}$ & 3.06 & 3.14 & 3.01 & $\mathrm{pc}$ & $11,11,3$ \\
\hline$Y_{\text {gal }}$ & 1.14 & 0.90 & 1.86 & $\mathrm{pc}$ & $11,11,3$ \\
\hline$Z_{\text {gal }}$ & -6.98 & -6.88 & -6.70 & $\mathrm{pc}$ & $11,11,3$ \\
\hline$\Delta_{\text {com }}$ & 0.05 & 0.24 & 0.77 & $\mathrm{pc}$ & $3,3,3$ \\
\hline$U$ & $-5.71 \pm 0.16$ & $-5.69 \pm 0.06$ & $-5.34 \pm 0.19$ & $\mathrm{~km} \mathrm{~s}^{-1}$ & $11,11,3$ \\
\hline$V$ & $-8.26 \pm 0.28$ & $-8.16 \pm 0.07$ & $-7.58 \pm 0.28$ & $\mathrm{~km} \mathrm{~s}^{-1}$ & $11,11,3$ \\
\hline$W$ & $-11.04 \pm 0.38$ & $-10.96 \pm 0.08$ & $-11.85 \pm 0.39$ & $\mathrm{~km} \mathrm{~s}^{-1}$ & $11,11,3$ \\
\hline$\Delta S$ & 0 & $0.13 \pm 0.51$ & $1.12 \pm 0.72$ & $\mathrm{~km} \mathrm{~s}^{-1}$ & 3,3 \\
\hline $\log \left(L / L_{\odot}\right)$ & $1.221 \pm 0.013$ & $-0.723 \pm 0.029$ & $-2.337 \pm 0.010$ & $\operatorname{dex}$ & $11,11,3$ \\
\hline Mass & $1.92 \pm 0.02$ & $0.73_{-0.01}^{+0.02}$ & $0.18 \pm 0.02$ & $M_{\odot}$ & $11,12,3$ \\
\hline$\theta$ & 0 & $7062^{\prime \prime} .7$ & $20407^{\prime \prime} 6$ & $\operatorname{arcsec}$ & 14,14 \\
\hline P.A. & 0 & 187.88 & 337.91 & deg & 14,14 \\
\hline
\end{tabular}

Notes. $\Delta_{\text {com }}$ is the approximate 3D separation between the star and the system's center of mass. $\Delta S$ is the difference in velocity compared to Fomalhaut A. Masses are estimated using evolutionary tracks and are not dynamically measured. $\theta$ is the projected angular separation from Fomalhaut, and P.A. is the position angle as measured north through east.

References. (1) van Leeuwen 2007 (distance = 1/parallax); (2) Roeser et al. 2010 (PPMXL); (3) this paper; (4) Gontcharov 2006; (5) Nordström et al. 2004; (6) Mermilliod \& Mermilliod 1994; (7) Busko \& Torres 1978; (8) Gray \& Garrison 1989 (standard); (9) Keenan \& McNeil 1989; (10) Scholz et al. 2005; (11) Mamajek 2012; (12) Casagrande et al. 2011; (13) Davis et al. 2005; (14) this paper; using positions from van Leeuwen (2007) and Roeser et al. (2010). In the table and throughout the paper, Galactic velocities $U$ and positions $X$ are defined toward the Galactic center, $V$ and $Y$ are in the direction of Galactic rotation, and $W$ and $Z$ are toward the north Galactic pole.

was truly associated with the Fomalhaut binary, with the main evidence being the coincidental proper motion and photometric distance. In this contribution, we combine newly determined accurate astrometric and radial velocity measurements for LP 876-10 to demonstrate that it too, like TW PsA, appears to be a distant companion of Fomalhaut, and should be considered "Fomalhaut C."

\section{ANALYSIS}

The stellar parameters for Fomalhaut, TW PsA, and LP 87610 are summarized in Table 1. Finder charts for LP 876-10 are provided in Figure 1.

\subsection{Photometry}

Adopted optical and infrared magnitudes from $0.4 \mu \mathrm{m}$ (B band) to $22 \mu \mathrm{m}$ (W4 band) are compiled in Table 2. $V$-band magnitudes of $12.618 \pm 0.012$ (Reid et al. 2003) and $12.62 \pm 0.02$ (UCAC4; Zacharias et al. 2013; Henden et al. 2012) have been previously reported for LP 876-10. Additionally, we measure Johnson $V=12.59 \pm 0.03$ based on three photometric nights of imaging with the Small and Moderate Aperture Research Telescope System's (SMARTS) $0.9 \mathrm{~m}$ telescope taken during 2004-2006, using 14" diameter aperture and the standards of
Landolt (1992, 2007). This measurement is consistent with preliminary values previously reported from this program (Bartlett 2007; Bartlett et al. 2007). Jao et al. (2005) and Winters et al. (2011) describe the REsearch Consortium On Nearby Stars (RECONS) ${ }^{12}$ photometry program. Pojmanski (1997) presents time series $V$-band ASAS photometry for this star (349 observations between UT 2000 November 22 and UT 2009 October 7 with quality flag A) with mean $V=12.62$ and $\mathrm{rms}$ scatter of $0.06 \mathrm{mag}$. The photometric errors of the individual measurements are typically $\sim 0.03 \mathrm{mag}$, so approximately $\sim 0.05$ mag of the scatter in the reported $V$ magnitudes appear to be due to intrinsic stellar variability. All of these previously mentioned $V$ magnitudes are calibrated to the Johnson system, either through Landolt standards (Reid et al. 2003; APASS/ UCAC4, RECONS) or Hipparcos (ASAS). The SuperWASP project took many photometric measurements of LP 876-10 in a $V$ band calibrated to the Tycho- $2 V_{T}$ photometric system (Pollacco et al. 2006; Butters et al. 2010; Høg et al. 2000). This photometry is later discussed in Section 2.8 for the purposes of measuring the rotation period, but was not included in our assessment of the mean Johnson $V$ magnitude. Based on photometry measured independently by Reid et al. (2003), Henden et al. (2012), Pojmanski (1997), and the RECONS observations with

\footnotetext{
12 www.recons.org
} 

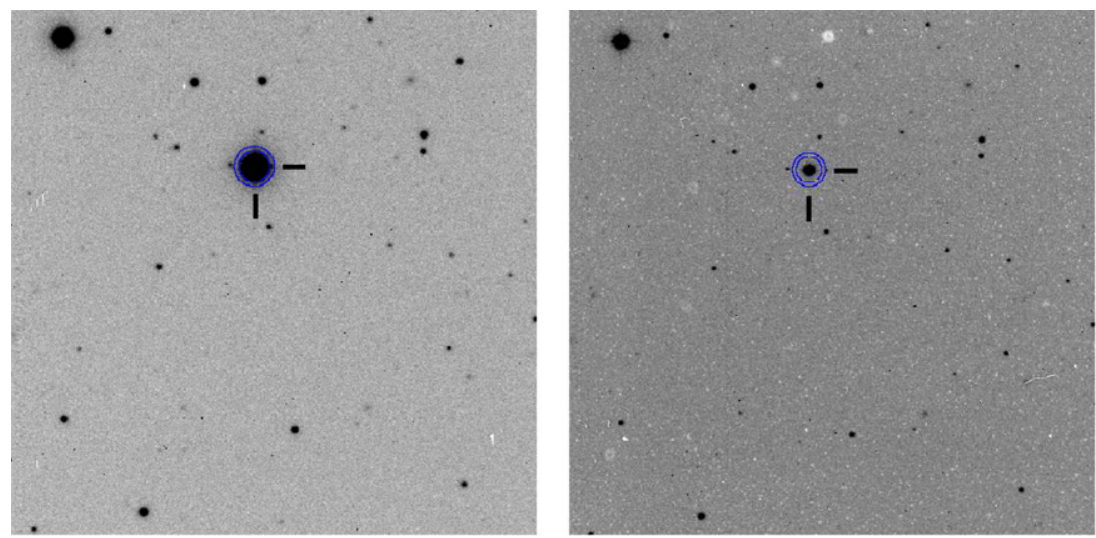

Figure 1. Left: Kron-Cousins $I$-band image of LP 876-10 taken with SMARTS $0.9 \mathrm{~m}$ telescope in 2004. Right: Johnson $V$-band image of LP 876-10 taken with the SMARTS $0.9 \mathrm{~m}$ telescope in 2012. The star is circled in blue and is near 22:48:04.5-24:22:08 (J2000). The field of view for both images is 6.8 arcmin square. North is up and east is to the left. Fomalhaut A and B are not in the field of view.

(A color version of this figure is available in the online journal.)

the SMARTS $0.9 \mathrm{~m}$ telescope, we adopt a mean $V$ magnitude of $12.62 \pm 0.01$ mag.

\subsection{Parallax and Proper Motion}

The parallax and proper motion of LP 876-10 have been measured during the long-term astrometry program carried out by RECONS at the SMARTS $0.9 \mathrm{~m}$ telescope. Jao et al. (2005) describes the astrometry program; however, we briefly summarize the program here. A filter is selected from the Johnson-Kron-Cousins $V R_{\mathrm{KC}} I_{\mathrm{KC}}$ filter set that provides a wellexposed reference field that, ideally, encircles the target star. Throughout the course of the observations, the same pointing (to within a few pixels) and filter are used. Centroids for the reference field and parallax star are extracted using SExtractor (Bertin \& Arnouts 1996) and corrected for differential color refraction using $V R_{\mathrm{KC}} I_{\mathrm{KC}}$ photometry of the reference and science target stars (see Section 2.1). Relative parallax and proper motion of the target star are solved for using the Gaussfit program. ${ }^{13}$ Correction from relative to absolute parallax is done by estimating the mean distance to the reference field stars, again, using $V R_{\mathrm{KC}} I_{\mathrm{KC}}$ photometry and the photometric distance relations of Henry et al. (2004).

LP 876-10 was included in the RECONS astrometric survey due to its close predicted photometric distance $(7.2 \pm 0.8 \mathrm{pc}$; Reid et al. 2003), which is consistent with preliminary parallax solutions from this program (Bartlett 2007; Bartlett et al. 2007). Based on 25 astrometric nights from 2004 to 2012, we derive an absolute trigonometric parallax of $132.07 \pm 1.19$ mas and a

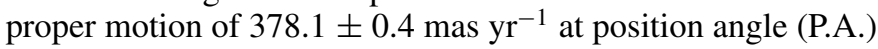
$118^{\circ} .0 \pm 0.1$ east of north. When the proper and parallactic motions are removed from the star's position, the residuals show no hint of curvature or any pattern that would suggest the existence of an unseen companion (see Section 2.4). At distance $d=7.57 \pm 0.07 \mathrm{pc}$, the three-dimensional (3D) separation of LP 876-10 from Fomalhaut is only $0.77 \pm 0.01 \mathrm{pc}\left(158_{-1}^{+2} \mathrm{kAU}\right)$, and from TW PsA it lies only $0.987_{-0.005}^{+0.006} \mathrm{pc}(203 \pm 1 \mathrm{kAU})$ away (Fomalhaut B; Mamajek 2012). Figure 2 summarizes the positions, separations, and proper motion vectors for Fomalhaut, TW PsA, and LP 876-10.

\footnotetext{
13 Available from the HST Astrometry Team at
} ftp://clyde.as.utexas.edu/pub/gaussfit/.

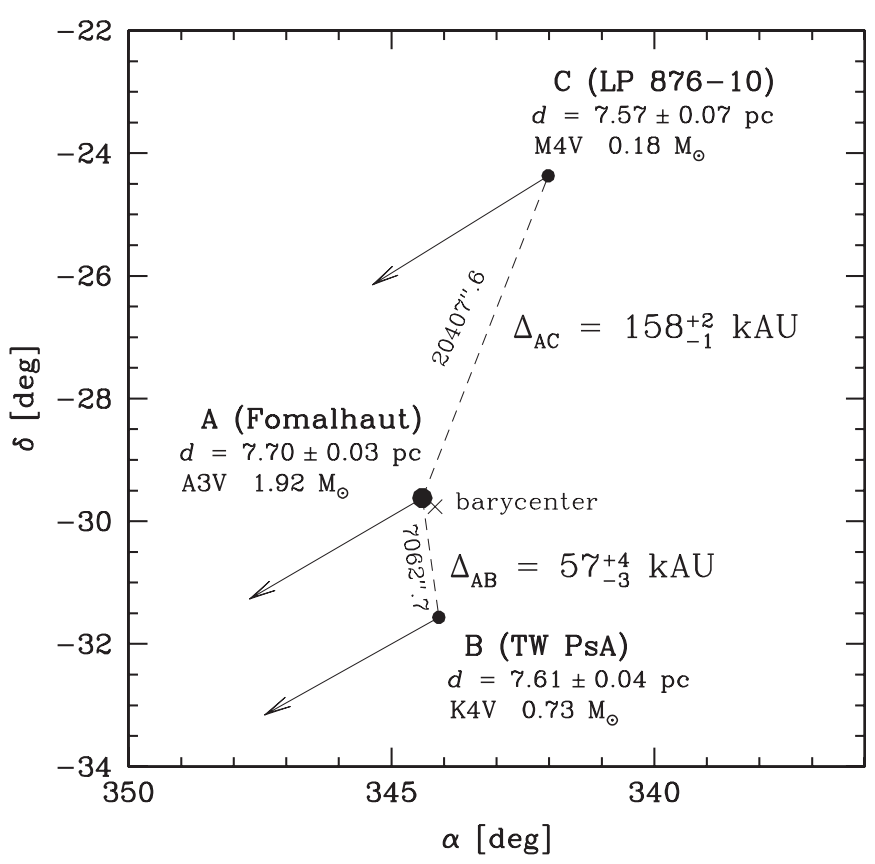

Figure 2. Positions and proper motion vectors for Fomalhaut A, B (TW PsA), and C (LP 876-10). The system barycenter is estimated to be at the position marked with an $\mathrm{X}$, at a distance of $7.67 \mathrm{pc}$ (more details are discussed in Section 2.2). The 3D separations $(\Delta)$ between $\mathrm{A}-\mathrm{B}$ and $\mathrm{A}-\mathrm{C}$ are listed in thousands of AU. The positions, parallaxes, distances, and proper motions are compiled in Table 1.

Blinking images suggest that the neighboring high proper motion star LP 876-11 could be a proper motion companion to LP 876-10; LP 876-11 is located 1'.8 away from LP 876-10 at $42^{\circ}$ east of north. However, we determine a proper motion

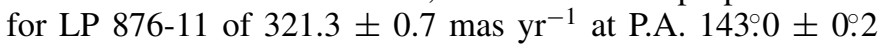
east of north, which is inconsistent with the measured motion for LP 876-10. Using 12 color-magnitude relations from Henry et al. (2004), we estimate a photometric distance to LP 876-11 of $730 \pm 120$ pc. We measure a trigonometric parallax of LP $876-11^{14}$ of $1 \pm 2$ mas, consistent with the photometric distance. We conclude that LP $876-11$ is not physically associated with LP 876-10.

\footnotetext{
14 Photometry for LP 876-11 from two nights of observations: $V=17.72$,
} $R_{\mathrm{KC}}=16.90$, and $I_{\mathrm{KC}}=16.04$. 
Table 2

LP 876-10 Photometry

\begin{tabular}{lcc}
\hline \hline$(1)$ & $(2)$ & $(3)$ \\
Band & Mag & Ref. \\
\hline$B$ & $14.31 \pm 0.01$ & 1,2 \\
$V$ & $12.62 \pm 0.01$ & 3 \\
$R_{\mathrm{KC}}$ & $11.31 \pm 0.03$ & 4 \\
$I_{\mathrm{KC}}$ & $9.61 \pm 0.03$ & 4 \\
$J$ & $8.075 \pm 0.023$ & 5 \\
$H$ & $7.527 \pm 0.055$ & 5 \\
$K_{s}$ & $7.206 \pm 0.021$ & 5 \\
$W 1$ & $6.911 \pm 0.034$ & 6 \\
$W 2$ & $6.803 \pm 0.022$ & 6 \\
$W 3$ & $6.705 \pm 0.016$ & 6 \\
$W 4$ & $6.497 \pm 0.058$ & 6
\end{tabular}

References. (1) Reid et al. (2003), (2) APASS photometry (Henden et al. 2012) reported in the UCAC4 catalog (Zacharias et al. 2013), (3) mean of the photometry from Reid et al. (2003), Henden et al. (2012), and Pojmanski (1997), and measured in this study using the SMARTS $0.9 \mathrm{~m}$ telescope; see the discussion in Section 2.1, (4) this paper, (5) Skrutskie et al. (2006; 2MASS PSC), and (6) Wright et al. (2010; WISE). The Johnson $B$ from APASS (Henden et al. 2012), and the Johnson $V$ and Kron-Cousins $R_{\mathrm{KC}} I_{\mathrm{KC}}$ photometry from RECONS (Jao et al. 2005; Winters et al. 2011), are all photometrically calibrated to Landolt (1992) standard stars.

\subsection{Radial Velocity}

A spectrum of LP 876-10 was taken with the CRIRES spectrograph on the $8.4 \mathrm{~m}$ Very Large Telescope UT1 (Antu) telescope on UT date 2009 June 16 as part of a near-infrared radial velocity survey of nearby late-type $M$ dwarfs (Bean et al. 2010). The CRIRES spectrum has wavelength coverage 2.292-2.349 $\mu \mathrm{m}$ over the effective $4096 \times 512$ focal plane detector, a mosaic of four Aladdin III InSb arrays (Kaeufl et al. 2004). The slit width was $0 ! 2$, yielding a resolving power of $R \simeq 100,000$ (resolution is $3 \mathrm{~km} \mathrm{~s}^{-1}$ at 2 pixel sampling). The signal-to-noise ratio $(\mathrm{S} / \mathrm{N})$ in the continuum of the spectrum was $\sim 170-220$. By fitting a broadened and shifted PHOENIX model spectrum from the Gaia Version 2.0 library (Hauschildt et al. 1999; Brott \& Hauschildt 2005) to the spectrum of LP 876-10, we determine a sizeable projected rotation velocity of $v \sin i=22 \pm 2 \mathrm{~km} \mathrm{~s}^{-1}$; a heliocentric radial velocity of $+6.5 \pm 0.5 \mathrm{~km} \mathrm{~s}^{-1}$ was also measured. Slit viewer images of LP 876-10 appear pointlike, and there is no sign of duplicity in the CRIRES spectrum. A more detailed spectroscopic analysis of LP 876-10 will be presented in a forthcoming paper (A. Seifahrt et al., in preparation).

\subsection{Duplicity}

While neither the astrometry nor the spectroscopy data are consistent with LP 876-10 being a binary, it is listed as a double star in the Washington Double Star catalog (WDS; Mason et al. $2001)^{15}$ as WDS 22481-2422 and with the discovery identifier "WSI 138."16 A single observation is reported for epoch 2010, with a reported companion at separation 0.'5 at P.A. $=144^{\circ}$,

\footnotetext{
15 Values are listed from the 2013 March 03 update of the WDS.

16 WSI = Washington Speckle Interferometer.
}

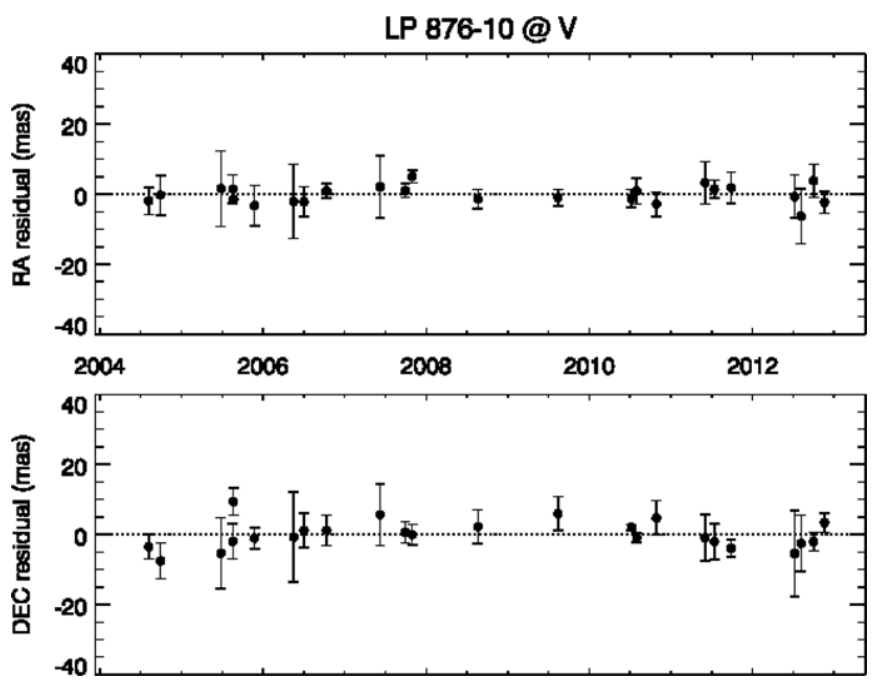

Figure 3. Astrometric residuals in R.A. (top) and decl. (bottom) for LP 876-10 in $V$-band images after subtracting the parallactic motion with $\varpi=$

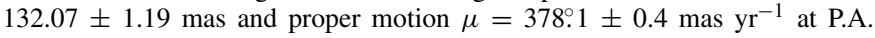
$118.0 \pm 0.1$ east of north. If the companion reported in the WDS (WSI $138 \mathrm{~B}$ ) were real, we predict that it would produce an astrometric perturbation on the photocenter at the $\sim 110$ mas level with a period of $\sim 13$ yr. Any perturbations due to unseen companions must be at the $<10$ mas level over the $\sim 8$ yr baseline.

with magnitudes of 12.80 and 14.80 (presumably $V$-band, as the combined magnitude (12.64) is similar to the adopted $V$ magnitude in Table 2). We are unable to confirm the existence of the companion reported in the WDS. In the 118 frames taken during 25 nights, with FWHMs in the range $1^{\prime \prime}$. 2 to 2 ". 8 , LP 87610 appeared to be a point source, with no evidence of elongation. With only a single observation, the possibility remains that the reported WDS companion may be a chance alignment between this high proper motion star and a background star (B. D. Mason 2013, private communication). However, we believe that a background star is unlikely to explain this discrepancy. Based on the UCAC4 position of LP 876-10 for epoch 2000.0 (Zacharias et al. 2013), the proper motion calculated in this paper, and the separation/P.A. value listed in the WDS, we estimate that the WSI 138 companion reported in the WDS had an approximate ICRS position 22:48:04.76-24:22:09.1 (epoch 2010). The only object listed in any Vizier-queryable catalog within $2^{\prime \prime}$ of this position is the WISE detection of LP 876-10 itself ( 0.5 away) during 2010. No plausible optical-IR counterpart within $2^{\prime \prime}$ of this position exists in the USNO-B1.0, SuperCOSMOS, GSC, and Two Micron All Sky Survey (2MASS) catalogs. It seems very unlikely that a bright $(V=14.8)$ background star can explain the faint companion to LP 876-10 reported in the WDS. If the companion was real and physically associated with LP 876-10, then its absolute magnitude $\left(M_{V}=15.40\right)$ would correspond to a $0.11 M_{\odot}$ star on the calibration of Delfosse et al. (2000). Given the projected separation $(0.5=3.8 \mathrm{AU})$, these values would predict an orbital period of $\sim 13.5 \mathrm{yr}$. Assuming zero eccentricity and face-on projection, one would predict an

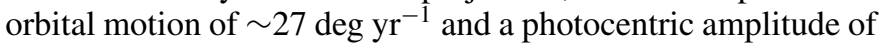
$\sim 110$ mas.

The predicted photocentric amplitude would be about half ( 50 mas over $8 \mathrm{yr}$ ) of the full amplitude ( 110 mas over $\sim 13.5 \mathrm{yr}$ ) during the observations to date. As seen in Figure 3, the astrometric solution using only parallax and proper motion is quite good, and any gravitational perturbations on LP 876-10 must be at the $<10$ mas level over $\sim 8$ yr, which easily rules out the predicted signal for the companion reported in the 
Table 3

Proper Motions for LP 876-10

\begin{tabular}{lcc}
\hline \hline $\begin{array}{l}\text { (1) } \\
\text { Reference }\end{array}$ & $\begin{array}{c}(2) \\
\mu_{\alpha} \\
\left(\mathrm{mas} \mathrm{yr}^{-1}\right)\end{array}$ & $\begin{array}{c}\mu_{\delta} \\
\left(\mathrm{mas}^{-1}\right)\end{array}$ \\
\hline Wroblewski \& Costa (1999) & $290 \pm 9$ & $-176 \pm 6$ \\
Hambly et al. (2001; SuperCOSMOS, UKST blue) & $326.9 \pm 15.66$ & $-191.9 \pm 19.21$ \\
Hambly et al. (2001; SuperCOSMOS, UKST red) & $329.9 \pm 13.12$ & $-187.6 \pm 14.10$ \\
Hambly et al. (2001; SuperCOSMOS, UKST IR) & $344.2 \pm 27.63$ & $-184.0 \pm 16.00$ \\
Monet et al. (2003; USNO-B1.0) & $312 \pm 2$ & $-180 \pm 3$ \\
Salim \& Gould (2003) & $323.3 \pm 5.5$ & $-174.8 \pm 5.5$ \\
Röser et al. (2008; PPMX) & $311.2 \pm 11.6$ & $-183.9 \pm 4.8$ \\
Roeser et al. (2010; PPMXL) & $322.6 \pm 4.8$ & $-181 \pm 8$ \\
Lépine \& Gaidos (2011) & $325 \pm 8$ & $-174.8 \pm 8$ \\
Zacharias et al. (2013; UCAC4) & $323.0 \pm 8$ & $-177.51 \pm 0.51$ \\
This paper & $333.84 \pm 0.51$ & \\
\hline
\end{tabular}

WDS. Table 3 shows that the differences between the long-term proper motions (e.g., SuperCOSMOS, USNO-B1.0, PPMX, and UCAC4) are largely within $\sim 5-10$ mas $\mathrm{yr}^{-1}$ (rms) of the $8 \mathrm{yr}$ baseline proper motion calculated in this survey, further suggesting that it would be difficult to hide a $\sim 50{\text { mas } \mathrm{yr}^{-1}}^{-1}$ perturbation of the photocentric motion. As the purported WDS companion should have a period only somewhat longer than the duration of our RECONS astrometric data set, and with a predicted photocentric amplitude similar in size to the observed parallax, we conclude that it is unlikely that the companion reported in the WDS catalog is real.

\subsection{Temperature, Luminosity, and Radius}

We estimated $T_{\text {eff }}$ for LP 876-10 by fitting the photometry in Table 2 to the BT-Settl grid of synthetic stellar spectra, which vary by effective temperature, metallicity, and surface gravity (Allard et al. 2012). Twenty-two colors consisting of combinations of the bands $V, R_{\mathrm{KC}}, I_{\mathrm{KC}}, J, H, K_{s}, W 1, W 2$, and $W 3$ were compared to grid interpolations based on models, and the best fit yielded an interpolated temperature of $T_{\text {eff }}=$ $3132 \mathrm{~K}$ and solar metallicity. We estimated the uncertainty in $T_{\text {eff }}$ due to metallicity and surface gravity by individually varying these parameters by one increment $(0.2 \mathrm{dex})$ and measuring the effect on the resultant $T_{\text {eff }}$. The uncertainty in the $T_{\text {eff }}$ breaks down approximately as follows: $\pm 33 \mathrm{~K}$ from the dispersion in color-based $T_{\text {eff }}$ estimates for the best fit, $\pm 50 \mathrm{~K}$ due to metallicity uncertainty, and $\pm 25 \mathrm{~K}$ due to uncertainty in $\log (g)$. Together this yields an overall $T_{\text {eff }}$ uncertainty of $\pm 65 \mathrm{~K}$. The systematic error due to the validity of the BT-Settl models is unknown; however, our derived $T_{\text {eff }}$ should be comparable to $\mathrm{M}$ dwarf $T_{\text {eff }}$ values derived using the same models (indeed Rajpurohit et al. 2013; similarly derives $T_{\text {eff }} \simeq 3100-3200 \mathrm{~K}$ for M4 dwarfs like LP 876-10 using BT-Settl models). The best-fitting BT-Settl synthetic spectrum had $T_{\text {eff }}=3100 \mathrm{~K}$, $[\mathrm{Fe} / \mathrm{H}]=0.0$, and $\log (g)=5.0$. From considerations of the star's color-magnitude diagram position (Section 2.6), we predict that LP 876-10 has a slightly subsolar metallicity, and lies near the zero-age main sequence for $\sim 0.2 M_{\odot}$ stars $(\log (g) \simeq 5.06$; Baraffe et al. 1998). The best-fitting BT-Settl synthetic spectrum is then adjusted via an iterative process to produce a match to the observed photometry. The process determines a small $\lambda$-dependent polynomial correction factor that is applied to the synthetic spectrum to cause small modifications in order to produce the best fit to the photometry (details of the technique are described in S. Dieterich et al. 2013, in preparation).
By directly integrating the spectral energy distribution made by fitting the photometry in Table 2 with solar composition BT-Settl models, we estimate $m_{\text {bol }}=9.994 \pm 0.020$, luminosity $=(1.763 \pm 0.042) \times 10^{31} \mathrm{erg} \mathrm{s}^{-1}, \log \left(L / L_{\odot}\right)=$ $-2.337 \pm 0.010$, absolute bolometric magnitude $M_{\mathrm{bol}}=$ $10.597 \pm 0.026$, and bolometric correction $\mathrm{BC}_{V}=m_{\text {bol }}-$ $V=-2.62 \pm 0.02$ (adopting solar parameters from Mamajek 2012; Pecaut \& Mamajek 2013). Combining this luminosity with our previous $T_{\text {eff }}$ estimate, we estimate a radius of $0.23 \pm 0.01 R_{\odot}$. Combined with our estimate of the projected rotation velocity $v \sin i\left(22 \pm 2 \mathrm{~km} \mathrm{~s}^{-1}\right)$, this places an upper limit on the rotation period of LP 876-10 of $0.55 \pm 0.05$ day (see Section 2.8).

\subsection{Color-Magnitude Diagram and Metallicity}

Using our new parallax and the photometry in Table 2, we estimate absolute magnitudes of $M_{V}=13.21 \pm 0.02$ and $M_{\mathrm{K}_{\mathrm{s}}}=7.81 \pm 0.03$. From Table 2 , we calculate a $\left(V-K_{s}\right)$ color of $5.40 \pm 0.02 \mathrm{mag}$. Using the $\left(V-K_{s}\right)$ versus $M_{V}$ relations from Henry et al. (2004) and Johnson \& Apps (2009), we predict photometric distances of $7.9 \pm 1.5 \mathrm{pc}$ and $7.7 \pm 1.4 \mathrm{pc}$, respectively, in excellent agreement with our trigonometric parallax distance. The agreement between the trigonometric parallax distance and the available photometric distances (Reid et al. 2003; this section) is also indicative that LP $876-10$ is unlikely to have an unresolved companion of similar mass, and it is more likely to be a main-sequence, rather than pre-mainsequence, star.

We can constrain the metallicity and age using the star's color-magnitude data. In Figure 4, we plot the star's $\left(V-K_{s}\right)$ color versus absolute magnitude $M_{V}$ and use the metallicity color-magnitude calibration of Johnson \& Apps (2009) to predict a metallicity of $[\mathrm{Fe} / \mathrm{H}]=-0.07 \mathrm{dex}$ (estimated accuracy \pm 0.06 dex). The calibration of Schlaufman \& Laughlin (2010) predicts a metallicity of $[\mathrm{Fe} / \mathrm{H}]=-0.15$ dex. These are in reasonable agreement with the high $\mathrm{S} / \mathrm{N}$ estimate for $\mathrm{TW}$ PsA (Fomalhaut B) from Barrado y Navascues et al. (1997) $([\mathrm{Fe} / \mathrm{H}]=-0.11 \pm 0.02)$. Other published $[\mathrm{Fe} / \mathrm{H}]$ estimates for TW PsA are $-0.01 \pm 0.09$ (Santos et al. 2004) and -0.20 (Morell 1994). Hence, both the photometric metallicity estimate for LP 876-10 and the spectroscopic metallicity estimates for TW PsA are self-consistent, and consistent with being very slightly subsolar $([\mathrm{Fe} / \mathrm{H}] \simeq-0.1 \mathrm{dex})$. 


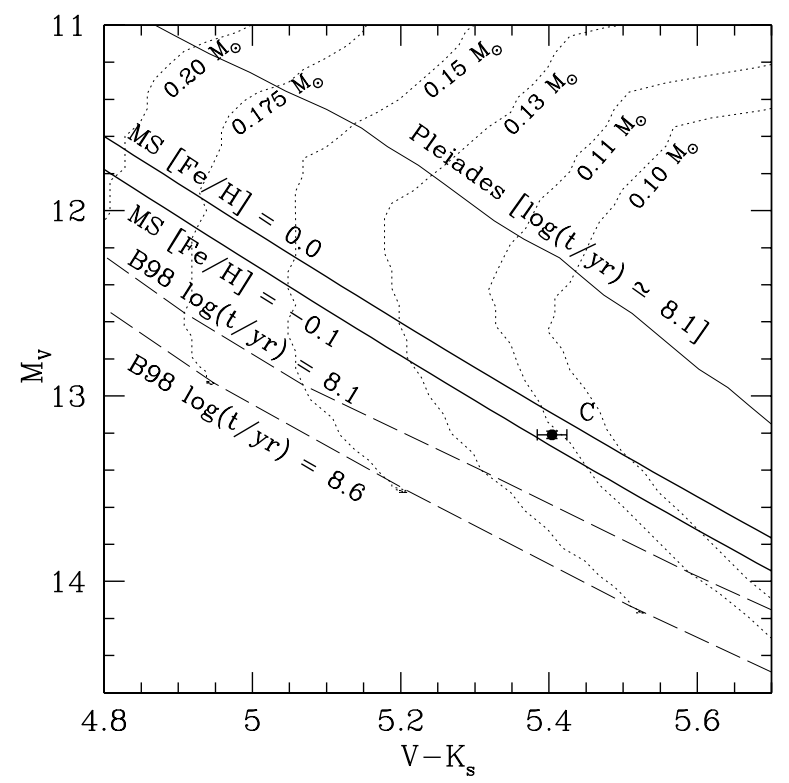

Figure 4. Color-magnitude (V-K vs. $M_{V}$ ) for Fomalhaut C (LP 876-10). The empirical main sequences for $[\mathrm{Fe} / \mathrm{H}]=0.0$ and -0.1 from Johnson \& Apps (2009) are plotted as thick solid lines, along with the color-magnitude sequence for the $\sim 125$ Myr old Pleiades cluster from Stauffer et al. (2007, adopting $d=133.5 \mathrm{pc}$ from Soderblom et al. 2005; thin solid line). The theoretical evolutionary tracks from Baraffe et al. (1998) are plotted as dotted lines, and the isochrones for the approximate age of the Pleiades $(\log (t / \mathrm{yr})=8.1)$ and Fomalhaut $\mathrm{A}$ and $\mathrm{B}(\log (t / \mathrm{yr})=8.6)$ are plotted as long dashed lines. The evolutionary tracks do not accurately predict the solar composition main sequence nor the Pleiades sequence for this color-magnitude combination. Note that the Pleiades has a well-determined lithium depletion boundary and mainsequence turn-off age consistent with $~ 125$ Myr (Ventura et al. 1998; Stauffer et al. 1998; Barrado y Navascués et al. 2004), and the main-sequence "turn-on" appears to be consistent with this age as well (Barenfeld et al. 2013). Fomalhaut C appears to lie on the empirical main sequence of Johnson \& Apps (2009) with $[\mathrm{Fe} / \mathrm{H}] \simeq-0.07$.

\subsection{Mass and Age Constraints}

Using the Delfosse et al. (2000) $M_{V}$ versus mass calibration for field $\mathrm{M}$ dwarfs (i.e., mixed metallicities and ages), the approximate mass of LP $876-10$ is $\sim 0.20 M_{\odot}$. Interpolating within the Baraffe et al. (1998) tracks, one finds that solar composition stars with masses of greater than $0.163 M_{\odot}$ are not ever predicted to be as faint as $M_{V}=13.21 \mathrm{mag}$ (see Figure 4). As the tracks are first and foremost tracing luminosity evolution as a function of mass and age, we also examine the constraints that the luminosity of LP 876-10 can provide. Through fitting BT-Settl models to the photometry, we estimate the luminosity to be $\log \left(L / L_{\odot}\right)=-2.337 \pm 0.010$ dex. We find that the Baraffe et al. (1998) and Dotter et al. (2008) solar composition tracks give essentially identical predictions that no stars with masses greater than $0.197 M_{\odot}$ are predicted to have luminosities this low. Using those tracks, we estimate that it takes a $0.2 M_{\odot}$ star $\sim 300$ Myr to reach within $\sim 0.01$ mag of the zero-age main sequence (the actual minimum in luminosity and radius occurs around $\sim 400-500 \mathrm{Myr}$ ). The appearance of LP 876-10 on the zero-age main sequence for $[\mathrm{M} / \mathrm{H}] \simeq-0.1$ is commensurate with the adopted age for Fomalhaut A and B (440 Myr; Mamajek 2012).

As seen in Figure 4, the Baraffe et al. (1998) isochrones do not accurately reproduce the empirical main sequence from Johnson \& Apps (2009) in this color regime, so our lower bound on the age of LP 876-10 is only approximate. Naively interpolating the mass and age of Fomalhaut $\mathrm{C}$ from the evolutionary tracks and isochrones would yield a mass of $\sim 0.11 M_{\odot}$ and an age of $\sim 60$ Myr. However, as can be seen in Figure 4, a $125 \mathrm{Myr}$ isochrone $(\log (\mathrm{age} / \mathrm{yr})=8.1)$ from the same tracks fails to replicate the intrinsic color-magnitude sequence for the 125 Myr old Pleiades (Barrado y Navascués et al. 2004). For the V-Ks color (5.4) of LP 876-10, the combination of the Pleiades color-magnitude sequence from Stauffer et al. (2007) and mean Pleiades distance from Soderblom et al. (2005) yields a Pleiades absolute magnitude of $M_{V}=12.24$. The Baraffe et al. (1998) isochrones for age $125 \mathrm{Myr}(\log (\mathrm{age} / \mathrm{yr})=8.1)$ predict absolute magnitude $M_{V}=13.57$ for $\mathrm{V}-\mathrm{K}_{s}=5.4,{ }^{17}$ i.e., 1.33 mag too faint! As summarized by Bell et al. (2012), "for all optical colors, no pre-main-sequence models follows the observed Pleiades sequence for temperatures cooler than 4000 K." Estimating isochronal ages using pre-main-sequence evolutionary tracks is quite problematic, with large systematic differences between tracks (see review by Soderblom 2010). For all of these reasons, we do not adopt the pre-main-sequence mass and isochronal age interpolated from the evolutionary tracks and isochrones in Figure 4, and instead constrain the age based on its proximity to the main sequence and infer the mass based on main-sequence absolute magnitude versus mass considerations. Given the empirical and theoretical constraints previously discussed, we adopt a mass of $0.18 \pm 0.02 M_{\odot}$ for Fomalhaut C.

\subsection{Rotation Period}

Photometric data from the online SuperWASP archive ${ }^{18}$ (Butters et al. 2010) consisting of 14,991 measurements for LP 87610 were extracted for two observing seasons (2007-2008). To search for a rotation period, we selected SuperWASP photometry from a single well-sampled season (2008) with $V_{\text {SuperWASP }}$ magnitudes between 12.46 and 12.70, with magnitude and photometric errors of less than $0.2 \mathrm{mag}$, and with a good TAMFLUX2 flag extraction. SuperWASP photometry is calibrated to the Tycho-2 $V_{T}$ system (Pollacco et al. 2006; Høg et al. 2000). There were 3162 points for subsequent analysis. To remove 1 day aliasing effects, all points during a single observing night were adjusted so that their average equaled the average seasonal magnitude of LP 876-10. A Lomb-Scargle (LS) periodogram with associated False Alarm Probabilities (FAPs) was calculated following Press et al. (1992), and the resultant periodogram is plotted in Figure 5. There is significant power $($ FAP $<0.001)$ seen in the LS periodogram of LP 876-10 at periods of $0.195,0.242,0.318$, and 0.466 days. A period of $P=0.466$ day would correspond to an equatorial velocity of $26 \mathrm{~km} \mathrm{~s}^{-1}$, which is only slightly larger than the observed $v \sin i$ ( $22 \mathrm{~km} \mathrm{~s}^{-1}$, corresponding to a maximum period of 0.55 day). For the star's mass and radius, we estimate a breakup velocity and period (following Townsend et al. 2004) of $386 \mathrm{~km} \mathrm{~s}^{-1}$ and $\mathrm{P}_{\text {breakup }}=0.03$ day, respectively. Hence, any of the periods between $\sim 0.03$ and $\sim 0.55$ day are possible, given the breakup and $v \sin i$ constraints, respectively. The fastest rotation period among 41 nearby field $\mathrm{M}$ dwarfs in the MEarth survey of Irwin et al. (2011) is 0.28 day. We test the robustness of the detection by injecting artificial sinusoidal $(P=0.466$ day) signals into a Gaussian distributed photometric data set with the same time cadence as the LP 876-10 data set. These tests indicate that the $0.195,0.242$, and 0.318 day peaks are aliasing effects due to the

\footnotetext{
17 The Baraffe et al. (1998) tracks use the CIT JHK photometric system. We convert the Baraffe et al. (1998) CIT photometry to 2MASS following Carpenter (2001).

18 http://www.wasp.le.ac.uk/public/
} 


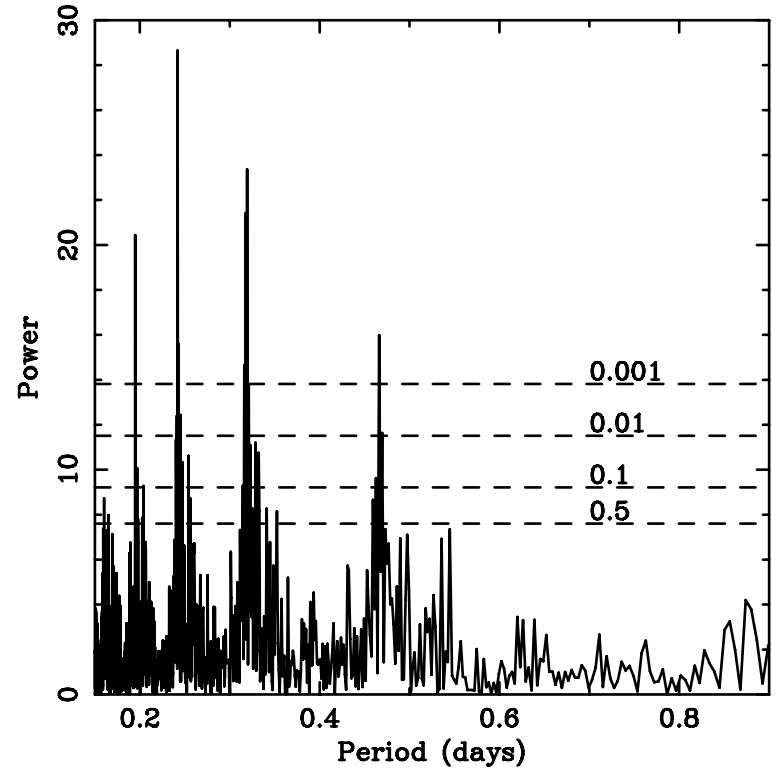

Figure 5. Lomb-Scargle periodogram for SuperWASP photometry for LP 876-10. The period at 0.466 day appears to be the real period, and tests indicate that the periods at 0.242 and 0.318 day are due to aliasing.

irregular time sampling of the light curve. We conclude that the $P=0.466$ day peak is most likely due to the rotation of the star.

Unfortunately, a rotation period of $\sim 0.5$ day for a $\sim 0.2 M_{\odot}$ star places negligible constraint on its age. Mid-M stars with rotation periods faster than 1 day are a nearly ubiquitous feature of stellar samples between ages of $\sim 2 \mathrm{Myr}$ and $\sim 10 \mathrm{Gyr}$ (see Figure 12 of Irwin et al. 2011). Figure 11 of Irwin et al. (2011) plots the masses of field $\mathrm{M}$ dwarfs versus their rotation periods measured by the MEarth survey. For stars of $\sim 0.2 M_{\odot}$, a rotation period of $\sim 0.466$ day is fast, however not unprecedented. Indeed, Irwin et al. (2011) finds that mid-M dwarfs like LP 876-10 can have periods of less than 1 day whether they are thin-disk or thick-disk stars. The survey of Irwin et al. (2011) had little difficulty finding kinematically old (>7 Gyr) thickdisk $M$ dwarfs with sub-day rotation periods. We conclude that attempts to age-date LP 876-10 via gyrochronology/rotation constraints appear fruitless.

\subsection{Activity}

Not only is LP 876-10 fast rotating, but, unsurprisingly, it appears to be a coronally active star as well. Voges et al. (1999) ranked LP $876-10^{19}$ as the most likely optical counterpart of the ROSAT All-Sky Survey (RASS) Bright Source Catalog (BSC) X-ray source 1RXS J224803.5-242240. The X-ray counterpart is $35^{\prime \prime}$ away from LP 876-10. However, the RASS BSC position error is $15^{\prime \prime}$, and LP 876-10 is the brightest optical source within $40^{\prime \prime}$, indicating that it is the likely X-ray source (Neuhaeuser et al. 1995). 1RXS J224803.5-242240 appears to be the brightest RASS X-ray source within a degree of LP 876-10. The fact that the position of the brightest RASS X-ray source within a degree of LP 876-10 lies within $40^{\prime \prime}$ of the rapidly rotating, nearby $M$ dwarf suggests to us that LP 876-10 is almost certainly the optical counterpart of 1RXS J224803.5-242240. The RASS BSC (Voges et al. 1999) reports a soft X-ray flux of 0.142 counts $\mathrm{s}^{-1}$ ( $28 \%$ uncertainty) with HR 1 hardness ratio of

\footnotetext{
${ }^{19}$ Listed under its Guide Star Catalog alias "GSC6964.01226."
}

$-0.23 \pm 0.21$, detected over a short exposure time of $176 \mathrm{~s}$. Using the energy conversion factor relation from Fleming et al. (1995), this translates to a coronal X-ray flux in the soft X-ray band (0.2-2.4 keV) of roughly $1.01 \times 10^{-12} \mathrm{erg} \mathrm{s}^{-1} \mathrm{~cm}^{-2}$. At $d=7.57 \mathrm{pc}$, this corresponds to an X-ray luminosity of $L_{X} \simeq$ $10^{27.84} \mathrm{erg} \mathrm{s}^{-1}$. This implies $\log \left(L_{X} / L_{\text {bol }}\right) \simeq-3.41$, i.e., a very active star close to X-ray saturation. This corroborates the very high projected rotational velocity measured spectroscopically ( $v \sin i=22 \mathrm{~km} \mathrm{~s}^{-1}$ ), which should induce strong magnetic activity.

\subsection{Velocity and Interloper Probability}

With our best measurements of the proper motion, radial velocity, and parallax, we calculate the $3 \mathrm{D}$ Galactic velocity of LP 876-10 to be $(U, V, W)=-5.3 \pm 0.2,-7.6 \pm 0.3$, $-11.9 \pm 0.4 \mathrm{~km} \mathrm{~s}^{-1}$. Comparing these values to those for Fomalhaut and Fomalhaut B (TW PsA), we find that LP 876-10's velocity only differs from that of Fomalhaut by $1.1 \pm 0.7 \mathrm{~km} \mathrm{~s}^{-1}$, and that of Fomalhaut B by $1.1 \pm 0.5 \mathrm{~km} \mathrm{~s}^{-1}$. Using the LSR velocity ellipsoid for both $\mathrm{dM}$ and dMe dwarfs estimated by Reid et al. (2002, their unweighted solution), and adopting the solar peculiar velocity with respect to the LSR from Schönrich et al. (2010), we naively only expect roughly 1 in $\sim 55,000$ field $\mathrm{M}$ dwarfs to have UVW velocities within $1.1 \mathrm{~km} \mathrm{~s}^{-1}$ of Fomalhaut, and roughly 1 in $\sim 12,000$ field $\mathrm{M}$ dwarfs to have a velocity within $2 \mathrm{~km} \mathrm{~s}^{-1}$.

Henry et al. (2006) report $239 \mathrm{M}$ dwarfs within $10 \mathrm{pc}$ that have accurate trigonometric parallaxes. These numbers are updated at recons.org, with a count as of 2012 January 1 of 248, which corresponds to a number density of $0.059 \mathrm{pc}^{-3}$. This space density implies that within a sphere of radius 1 pc surrounding Fomalhaut, we would expect to find $0.25 \mathrm{M}$ dwarfs. Hence, we estimate the probability that a random $\mathrm{M}$ dwarf could appear within $1 \mathrm{pc}$ of Fomalhaut, and sharing its velocity within less than $2 \mathrm{~km} \mathrm{~s}^{-1}$, as approximately 1 in $\sim 10^{4.7}$ (and sharing its velocity within less than $1.1 \mathrm{~km} \mathrm{~s}^{-1}$ as roughly 1 in $\sim 10^{5.3}$ ). For comparison, one would expect to have to encircle a sphere $\sim 36 \mathrm{pc}$ in radius in the local Galactic disk in order to find another M dwarf whose motion randomly agreed with that of Fomalhaut within less than $2 \mathrm{~km} \mathrm{~s}^{-1}$. Our probability estimates do not take into account the similarity in the spectroscopic metallicity of TW PsA and the photometric metallicity of LP 876-10, which provides further agreement. We conclude that LP 876-10 appears to be related to Fomalhaut and TW PsA beyond a reasonable doubt.

\subsection{The Castor Moving Group}

Fomalhaut was listed by Barrado y Navascues (1998) as a potential member of the Castor Moving Group (CMG). The comotion of LP 876-10 with Fomalhaut may be less significant if Fomalhaut is immersed in a swarm of co-moving stars like the purported CMG. The origin and nature of moving groups like the CMG is an active field of study (e.g., Famaey et al. 2005; Murgas et al. 2013). That the CMG represents a kinematic group of stars of common age and birthsite is unlikely.

Calculating revised space motions for the $14 \mathrm{CMG}$ "members" ("Y" or "Y?" members) from Barrado y Navascues (1998), using revised Hipparcos astrometry (van Leeuwen 2007) and the best available radial velocities (Barbier-Brossat \& Figon 2000; Gontcharov 2006), we find that the CMG stars have a median velocity of $(U, V, W)=-11.1 \pm 1.9,-8.6 \pm 0.8$, $-9.7 \pm 1.0 \mathrm{~km} \mathrm{~s}^{-1}$, with standard deviations of $6.1,3.6$, and 
$4.2 \mathrm{~km} \mathrm{~s}^{-1}$. The scatters are much larger than the typical velocity errors, and larger than the one-dimensional velocity dispersions of nearby clusters and associations $\left(<1.5 \mathrm{~km} \mathrm{~s}^{-1}\right.$; Madsen et al. 2002; Mamajek 2010). The velocity for Fomalhaut differs from the CMG median velocity by $5.6 \pm 2.3 \mathrm{~km} \mathrm{~s}^{-1}$. The list of "final" members in Barrado y Navascues (1998) comprises $\sim 27 M_{\odot}$ of stars spread out over a volume of $\sim 55,000 \mathrm{pc}^{3}$, implying that the density of CMG members in the solar neighborhood is roughly $\sim 0.004 \times$ the local disk density $\left(0.12 M_{\odot} \mathrm{pc}^{-3}\right.$; van Leeuwen 2007). The stellar systems in the CMG have negligible interaction with one another, and so their motions are completely dominated by the local Galactic potential.

The velocity differences between Fomalhaut and individual CMG members are illuminating, and we discuss the famous CMG members Vega, LP 944-20, and Castor itself, in more detail. Vega is a proposed fellow CMG star of either similar age (455 \pm 13 Myr; Yoon et al. 2010) or somewhat older age $\left(700_{-75}^{+150}\right.$ Myr; Monnier et al. 2012) than that of Fomalhaut (440 \pm 40 Myr; Mamajek 2012). Could Vega and Fomalhaut be related? Using the revised Hipparcos astrometry for Vega and its mean radial velocity reported by Parthasarathy \& Lambert (1987), we estimate for Vega a velocity of $(U, V, W)=$ $-15.9 \pm 0.7,-6.2 \pm 0.5,-7.7 \pm 0.3 \mathrm{~km} \mathrm{~s}^{-1}$. Vega's velocity differs from that of Fomalhaut by $10.9 \pm 1.0 \mathrm{~km} \mathrm{~s}^{-1}$, and only $10 \mathrm{Myr}$ ago their separations differed by $\sim 110 \pm 10 \mathrm{pc}$. Another nearby famous CMG "member" is the nearby candidate brown dwarf LP 944-2020 (Ribas 2003). Adopting the astrometry from Tinney (1996) and a mean radial velocity of $+9.0 \pm 0.5 \mathrm{~km} \mathrm{~s}^{-1}$ (based on measurements from Martín et al. 2006), we calculate a velocity for LP 944-20 of $(U, V, W)=-12.2 \pm 0.4,-5.6 \pm 0.3$, $-2.8 \pm 0.3 \mathrm{~km} \mathrm{~s}^{-1}$. LP 944-20 is currently situated $6.6 \mathrm{pc}$ away from Fomalhaut, and its velocity differs from that of Fomalhaut by $10.9 \pm 0.8 \mathrm{~km} \mathrm{~s}^{-1}$. Only $10 \mathrm{Myr}$ ago, LP 94420 and Fomalhaut were separated by $\sim 100 \pm 8 \mathrm{pc}$, and were only more widely separated in the past. We also investigated whether there was any association between Fomalhaut and Castor itself. For the Castor sextuplet system, we adopt the recent parallax estimate from Torres \& Ribas (2002), the long-term system proper motion from PPMX (Röser et al. 2008), and the center-of-mass radial velocity estimate from Heintz (1988; we adopt a radial velocity uncertainty of $1 \mathrm{~km} \mathrm{~s}^{-1}$ in our calculations). These values are consistent with the Castor system having a velocity of $(U, V, W)=-7.5 \pm 0.7,-3.7 \pm 0.6$, $-11.5 \pm 0.4 \mathrm{~km} \mathrm{~s}^{-1}$. Fomalhaut is currently $\sim 21 \mathrm{pc}$ away from the Castor system, differing in velocity by a significant margin $\left(4.9 \pm 1.1 \mathrm{~km} \mathrm{~s}^{-1}\right)$, and only $10 \mathrm{Myr}$ ago Fomalhaut and Castor were separated by $\sim 50 \pm 5 \mathrm{pc}$ and were even more distant in the past (more than 700 pc 100 Myr ago).

Despite these stars (the Fomalhaut system, Vega, LP 944-20, and the Castor system) being young and having somewhat similar velocities, their velocities are well-constrained enough and different enough that it is clear that they were not in the vicinity of one another even in the recent past, let alone a couple of Galactic orbits ago. We conclude that the CMG is comprised of stars from different birthsites rather than a coeval system, and hence "membership" to the CMG does not provide useful age constraints for the Fomalhaut system (or Vega, LP 944-20, Castor, or other CMG members).

\footnotetext{
20 A new RECONS parallax has been measured which places LP 944-20 at a distance of $6.4 \mathrm{pc}$ (Dieterich et al., submitted), making it most likely a star near the H-burning limit rather than a brown dwarf. The new distance revises LP 944-20's space motion to $(U, V, W)=-14.9,-5.9,-1.5 \mathrm{~km} \mathrm{~s}^{-1}$, which differs from that of Fomalhaut by $13.5 \mathrm{~km} \mathrm{~s}^{-1}$, and does not change the qualitative conclusions in the text.
}

\subsection{A Bound Companion?}

One predicts that stellar companions in multiple systems can exist with separations up to their tidal (Jacobi) radius with respect to the Galactic potential. Jiang \& Tremaine (2010) parameterize the tidal radius $r_{t}$ as:

$$
r_{\mathrm{t}}=\left\{\frac{G\left(M_{1}+M_{2}\right)}{4 \Omega A}\right\}^{1 / 3},
$$

where $G$ is the Newtonian gravitational constant, $M_{1}$ and $M_{2}$ are the masses of the stars, $\Omega$ is the Galactic angular circular speed (orbital velocity divided by Galactocentric radius), and $A$ is the Oort parameter. Adopting modern estimates of the relevant Galactic parameters, and rewriting the expression from Jiang \& Tremaine (2010), we estimate the tidal radius to be:

$$
r_{\mathrm{t}}=1.35 \mathrm{pc}\left\{\frac{M_{\text {total }}}{M_{\odot}}\right\}^{1 / 3}
$$

Summing the masses of the Fomalhaut system components $\left(2.83 M_{\odot}\right)$, one predicts a tidal radius of $\sim 1.9$ pc. The tidal radius for Fomalhaut $\mathrm{A}$ alone is $\sim 1.7 \mathrm{pc}$. Hence, the separation of $\sim 0.8 \mathrm{pc}$ between Fomalhaut $\mathrm{C}$ and $\mathrm{A}$ is not dynamically implausible for a bound system. Recent systematic surveys for wide-separation pairs using modern astrometric databases have started to yield many previously unrecognized parsec-scale common proper motion pairs (e.g., Caballero 2010; Shaya \& Olling 2011), making Fomalhaut C less unusual than it may have appeared even a decade ago. Stable orbits for timescales longer than a Gyr are also possible for separations larger than the tidal radius if the distant companion orbits retrograde to the Galactic rotation (e.g., Makarov 2012). Indeed, higher-precision radial velocities for the components of the Fomalhaut system and taking into account the sub-kilometer second $^{-1}$ effects of convective blueshift and gravitational redshift may lend themselves to providing a test as to whether Fomalhaut $\mathrm{C}$ is orbiting Fomalhaut $\mathrm{AB}$ either retrograde or prograde to the Galactic rotation (V. Makarov 2013, private communication).

Fomalhaut $\mathrm{A}$ and $\mathrm{B}$ are separated by $\Delta_{A B}=57.4_{-2.5}^{+3.9} \mathrm{kAU}$, and Fomalhaut $\mathrm{C}$ is separated by $\Delta_{A C}=158.2_{-1.2}^{+2.3} \mathrm{kAU}$ from $\mathrm{A}$ and by $\Delta_{B C}=203.4_{-0.8}^{+1.0} \mathrm{kAU}$ from $\mathrm{B}$. We calculate the position of the barycenter (center of mass) for the system using the Galactic $(X, Y, Z)$ positions and masses in Table $1:(X, Y, Z)_{\mathrm{com}}=3.08$, $1.13,-6.93 \mathrm{pc}$. Converting this position to the equatorial ICRS coordinate system yields $(\alpha, \delta)=344.179,-29.792$, at a distance of $7.67 \mathrm{pc}$. We can make a rough estimate of the orbital period of $\mathrm{C}$ around the $\mathrm{AB}$ pair. $\mathrm{C}$ is currently located $\sim 0.77 \mathrm{pc}$ from the system's center of mass. If $\mathrm{C}$ is currently near apastron (not an unreasonable assumption given that binary stars will spend most of their time near apastron), and if C's periastron must almost certainly be larger than B's current separation from the system barycenter $(0.24 \mathrm{pc})$, then a reasonable first estimate of C's orbit is $a \sim 0.5 \mathrm{pc}$ and $e \sim 0.5$. For the total mass of the Fomalhaut system $\left(2.83 M_{\odot}\right)$, this translates to an approximate orbital period of $\sim 20 \mathrm{Myr}$, or $\sim 5 \%$ of the system's age. The predicted orbital velocity of LP 876-10 around the Fomalhaut system barycenter would be $\sim 0.15 \mathrm{~km} \mathrm{~s}^{-1}$. Given the masses and configuration of the $\mathrm{AB}$ pair, the escape velocity of $\mathrm{C}$ is $\sim 0.2 \mathrm{~km} \mathrm{~s}^{-1}$.

How stable is Fomalhaut C's orbit with respect to A and B? Obviously, the orbit of $\mathrm{AB}$ and $\mathrm{AB}-\mathrm{C}$ are not well constrained. 
We only have fairly accurate estimates of the relevant mass ratios and current separations, while the semimajor axes and eccentricities are unknown. The mass of $\mathrm{C}$ is very small compared to that for the $\mathrm{AB}$ pair $\left(\mu=M_{C} /\left(M_{A}+M_{B}\right) \simeq\right.$ 0.07 ), and its current separation from the center of mass for the system is approximately $159 \mathrm{kAU}$. Based on simulations of test particles in the vicinity of binary systems of varying semimajor axis, mass ratio, and eccentricity, Holman \& Wiegert (1999) provided estimates of the widest stable orbit around a member of a binary system (S-type orbits), and the closest orbit around both members of a binary system (P-type orbits). If the current $\mathrm{A}-\mathrm{B}$ separation is equivalent to its semimajor axis (assume $e=$ $0 ; a=57.4 \mathrm{kAU})$, then the minimum stable semimajor axis for $\mathrm{C}$ is predicted to be $\sim 135 \mathrm{kAU}$. Tokovinin (1998) estimates that the mean eccentricity for wide binary pairs is $\langle e\rangle \simeq 2 / 3$. If Fomalhaut B is currently near apastron with $e \sim 2 / 3$, then $a \sim$ $34 \mathrm{kAU}$ and the minimum stable semimajor axis for Fomalhaut $\mathrm{C}$ is $\sim 140 \mathrm{kAU}$. There are plausible ranges of orbital parameters for Fomalhaut B and $\mathrm{C}$ that would be dynamically stable over many orbits.

Could LP 876-10 be genetically related to Fomalhaut AB, but we are "catching it in the act" of being an unbound escapee of the Fomalhaut system? We argue that this is very unlikely. LP 876-10 has a velocity statistically consistent with that of Fomalhaut $\mathrm{A}$ and $\mathrm{B}\left(\Delta S=1.1 \pm 0.7 \mathrm{~km} \mathrm{~s}^{-1}\right)$. If the star actually had a velocity difference of $>0.2 \mathrm{~km} \mathrm{~s}^{-1}$ (i.e., above escape velocity), with respect to the Fomalhaut $\mathrm{AB}$ barycenter, then it would not spend much time in the vicinity of Fomalhaut or near its tidal radius. The approximate timescale that LP 876-10 would spend within Fomalhaut's tidal radius is approximately $t \simeq r_{t} / \Delta S$, where $r_{t} \simeq 1.9 \mathrm{pc}$ is the tidal radius of Fomalhaut $\mathrm{AB}$, and we posit that $v$ must be larger than the escape velocity $\left(0.2 \mathrm{~km} \mathrm{~s}^{-1}=0.2 \mathrm{pc} \mathrm{Myr}^{-1}\right)$. Hence,

$$
t_{\mathrm{Myr}} \simeq \frac{r_{t}}{\Delta S}<9.5 \mathrm{Myr}
$$

For $\Delta S \sim v_{\text {esc }} \sim 0.2 \mathrm{~km} \mathrm{~s}^{-1}$, LP 876-10 could spend of the order of $\sim 10$ Myr within the tidal radius of Fomalhaut. For a velocity difference of $\sim 1 \mathrm{~km} \mathrm{~s}^{-1}$, LP 876-10 would spend only $\sim 2$ Myr. Velocity differences between LP 876-10 and Fomalhaut of $\Delta S$ greater than $2.5 \mathrm{~km} \mathrm{~s}^{-1}$ are ruled out at 95\% confidence, so timescales for LP 876-10 being unbound and within the tidal radius of Fomalhaut shorter than $\sim 0.8 \mathrm{Myr}$ are ruled out. Hence, $t$ would have to be of the order of $\sim 1-10 \mathrm{Myr}$ if LP 876-10 is unbound to Fomalhaut AB. For a main-sequence lifetime of Fomalhaut A of $\sim 0.9 \mathrm{Gyr}$, this suggests that for LP $876-10$ to be an unbound member of the Fomalhaut system in a state of disintegration, then we would have to be witnesses to an unusual dynamical state predicted to occur over $\sim 0.01 \%-0.1 \%$ over the lifetime of Fomalhaut $A$. This seems rather unlikely, and the simplest explanation for the agreement in velocities at the kilometer-per-second level between LP 876-10 and Fomalhaut $\mathrm{A}$ and $\mathrm{B}$, and its position within the tidal radius of Fomalhaut $\mathrm{AB}$, is that LP 876-10 is a third bound component of the Fomalhaut system.

\section{SUMMARY}

LP 876-10 is an active $\left(\log \left(L_{\mathrm{X}} / L_{\text {bol }}\right) \simeq-3.4\right)$, fast-rotating ( $P \simeq 0.47$ day) star lying within $1 \mathrm{pc}$ of Fomalhaut and TW PsA (Fomalhaut B), and sharing their motion within $\sim 1 \mathrm{~km} \mathrm{~s}^{-1}$. Mamajek (2012) showed that the isochronal age of Fomalhaut and various age diagnostics for TW PsA (rotation, X-ray emission, and $\mathrm{Li}$ abundance) were consistent with an age of $440 \pm 40$ Myr for the pair. The appearance of LP 876-10 on the main sequence hints that it is $>300 \mathrm{Myr}$ in age, and its photometric metallicity $([\mathrm{Fe} / \mathrm{H}] \simeq-0.1)$ is in good agreement with spectroscopic metallicity estimates for TW PsA. We argue that the purported membership of the Fomalhaut system to the CMG does not provide a useful age constraint on the system.

Based on its position, velocity, and color-magnitude data, we argue that LP 876-10 is a third stellar component in the Fomalhaut system. The chances of an interloper field $\mathrm{M}$ dwarf sharing the velocity of Fomalhaut within $1 \mathrm{~km} \mathrm{~s}^{-1}$ and lying within $1 \mathrm{pc}$ of Fomalhaut is $<10^{-5}$, hence LP $876-10$ is almost certainly physically related to Fomalhaut $\mathrm{A}$ and $\mathrm{B}$. The chances that we are catching the Fomalhaut system in a state of disintegration, where LP 876-10 is currently escaping with velocity greater than its predicted escape velocity $\left(0.2 \mathrm{~km} \mathrm{~s}^{-1}\right)$, is statistically unlikely $\left(<10^{-3}\right)$. Hence, we argue that LP $876-10$ is most likely a bound low-mass stellar companion to the Fomalhaut system, which has a well-determined age of $440 \pm 40 \mathrm{Myr}$ (Mamajek 2012). This makes the previously barely studied M dwarf LP 876-10 ("Fomalhaut C"), only recently added to the census of stars within $10 \mathrm{pc}$ via the RECONS astrometry program, one of the few red dwarfs in the solar neighborhood with a strongly constrained $(\sim 10 \%)$ age and metallicity $([\mathrm{Fe} / \mathrm{H}] \simeq-0.1)$. Given the difficulty in calibrating the age and metallicity scale for $\mathrm{M}$ dwarfs, Fomalhaut $\mathrm{C}$ provides a useful anchor among the mid-M stars, and another rare example of a low-mass companion with separation approaching a parsec. The existence of both Fomalhaut C (LP 876-10) and B (TW PsA) should be considered for future dynamical calculations trying to explain the unusual offset $(\sim 13 \mathrm{AU})$ between Fomalhaut A and its debris disk (Kalas et al. 2005) and the eccentric orbit for the planet candidate Fomalhaut Ab (Kalas et al. 2013).

We thank the referee for a prompt and thoughtful review, which significantly improved the paper. We thank Brian Mason, Mark Pecaut, Valeri Makarov, Massimo Marengo, John Bangert, Christine Hackman, Demetrios Matsakis, Sean Urban, Alice Quillen, and Paul Kalas for discussions on LP 876-10, Fomalhaut, and comments on the paper. E.E.M. acknowledges support from NSF award AST-1008908. J.L.B. acknowledges support from the University of Virginia, Hampden-Sydney College, and the Levinson Fund of the Peninsula Community Foundation. The RECONS effort is supported primarily by the National Science Foundation through grants AST 05-07711 and AST 09-08402. Observations were initially made possible by NOAO's Survey Program and have continued via the SMARTS Consortium. This research has made use of NASA ADS, SIMBAD, Vizier, and data products from 2MASS, WISE, UCAC, SuperWASP, ASAS, and Hipparcos. This research was made possible through the use of the AAVSO Photometric All-Sky Survey (APASS), funded by the Robert Martin Ayers Sciences Fund. This research has made use of the Washington Double Star Catalog maintained at the U.S. Naval Observatory.

Note added. T. Forveille (2013, private communication) reports that "CFHT adaptive optics images at two epochs (2004 July and 2005 October) show no companion (to LP 876-10)," consistent with the results discussed in Section 2.4.

\section{REFERENCES}

Allard, F., Homeier, D., Freytag, B., \& Sharp, C. M. 2012, EAS Publ. Ser, 57,3

Allen, R. H. 1963, Star Names: Their Lore and Meaning (New York: Dover) 
Baraffe, I., Chabrier, G., Allard, F., \& Hauschildt, P. H. 1998, A\&A, 337, 403 Barbier-Brossat, M., \& Figon, P. 2000, A\&AS, 142, 217

Barenfeld, S. A., Bubar, E. J., Mamajek, E. E., \& Young, P. A. 2013, ApJ, 766, 6

Barrado y Navascues, D. 1998, A\&A, 339, 831

Barrado y Navascues, D., Stauffer, J. R., Hartmann, L., \& Balachandran, S. C. 1997, ApJ, 475, 313

Barrado y Navascués, D., Stauffer, J. R., \& Jayawardhana, R. 2004, ApJ, 614,386

Bartlett, J. L. 2007, PhD thesis, Univ. Virginia

Bartlett, J. L., Ianna, P. A., Henry, T. J., et al. 2007, BAAS, 39, 772

Bean, J. L., Seifahrt, A., Hartman, H., et al. 2010, ApJ, 713, 410

Bell, C. P. M., Naylor, T., Mayne, N. J., Jeffries, R. D., \& Littlefair, S. P. 2012, MNRAS, 424, 3178

Bertin, E., \& Arnouts, S. 1996, A\&AS, 117, 393

Brott, I., \& Hauschildt, P. H. 2005, in The Three-Dimensional Universe with Gaia, ed. C. Turon, K. S. O’Flaherty, \& M. A. C. Perryman (ESA SP, Vol. 576; Noordwijk: ESA), 565

Burnham, R., Jr. 1978, Burnham's Celestial Handbook, Vols. 1, 2, and 3 (New York: Dover)

Burnham, S. W. 1906, A General Catalogue of Double Stars Within $121^{\circ}$ of the North Pole (Washington, DC: The Carnegie Institution of Washington)

Busko, I. C., \& Torres, C. A. O. 1978, A\&A, 64, 153

Butters, O. W., West, R. G., Anderson, D. R., et al. 2010, A\&A, 520, L10

Caballero, J. A. 2010, A\&A, 514, A98

Carpenter, J. M. 2001, AJ, 121, 2851

Casagrande, L., Schönrich, R., Asplund, M., et al. 2011, A\&A, 530, A138

Currie, T., Debes, J., Rodigas, T. J., et al. 2012, ApJL, 760, L32

Davis, J., Richichi, A., Ballester, P., et al. 2005, AN, 326, 25

Delfosse, X., Forveille, T., Ségransan, D., et al. 2000, A\&A, 364, 217

Dotter, A., Chaboyer, B., Jevremović, D., et al. 2008, ApJS, 178, 89

Famaey, B., Jorissen, A., Luri, X., et al. 2005, A\&A, 430, 165

Fleming, T. A., Schmitt, J. H. M. M., \& Giampapa, M. S. 1995, ApJ, 450,401

Gillett, F. C. 1986, in Light on Dark Matter, Vol. 124, ed. F. P. Israel (Dordrecht: Reidel), 61

Gontcharov, G. A. 2006, AstL, 32, 759

Gray, R. O., \& Garrison, R. F. 1989, ApJS, 70, 623

Hambly, N. C., MacGillivray, H. T., Read, M. A., et al. 2001, MNRAS, 326, 1279 (SuperCOSMOS)

Hauschildt, P. H., Allard, F., \& Baron, E. 1999, ApJ, 512, 377

Heintz, W. D. 1988, PASP, 100, 834

Henden, A. A., Levine, S. E., Terrell, D., Smith, T. C., \& Welch, D. 2012, JAVSO, 40, 430

Henry, T. J., Jao, W.-C., Subasavage, J. P., et al. 2006, AJ, 132, 2360

Henry, T. J., Subasavage, J. P., Brown, M. A., et al. 2004, AJ, 128, 2460

Høg, E., Fabricius, C., Makarov, V. V., et al. 2000, A\&A, 355, L27

Holman, M. J., \& Wiegert, P. A. 1999, AJ, 117, 621

Irwin, J., Berta, Z. K., Burke, C. J., et al. 2011, ApJ, 727, 56

Jao, W.-C., Henry, T. J., Subasavage, J. P., et al. 2005, AJ, 129, 1954

Jiang, Y.-F., \& Tremaine, S. 2010, MNRAS, 401, 977

Johnson, J. A., \& Apps, K. 2009, ApJ, 699, 933

Kaeufl, H.-U., Ballester, P., Biereichel, P., et al. 2004, Proc. SPIE, 5492, 1218

Kalas, P., Graham, J. R., Chiang, E., et al. 2008, Sci, 322, 1345

Kalas, P., Graham, J. R., \& Clampin, M. 2005, Natur, 435, 1067

Kalas, P., Graham, J. R., Fitzgerald, M. P., \& Clampin, M. 2013, ApJ, 775, 56

Keenan, P. C., \& McNeil, R. C. 1989, ApJS, 71, 245

Landolt, A. U. 1992, AJ, 104, 340

Landolt, A. U. 2007, AJ, 133, 2502

Lépine, S., \& Gaidos, E. 2011, AJ, 142, 138

Luyten, W. J. 1938, AJ, 47, 115
Luyten, W. J., \& Hughes, H. S. 1980, Proper Motion Survey with the Forty-Eight Inch Schmidt Telescope. LV. First Supplement to the NLTT Catalogue, Vol. 55 (Minneapolis, MN: Univ. Minnesota), 1

Madsen, S., Dravins, D., \& Lindegren, L. 2002, A\&A, 381, 446

Makarov, V. V. 2012, MNRAS, 421, L11

Mamajek, E. E. 2010, BAAS, 42, 455.05

Mamajek, E. E. 2012, ApJL, 754, L20

Marengo, M., Stapelfeldt, K., Werner, M. W., et al. 2009, ApJ, 700, 1647

Martín, E. L., Guenther, E., Zapatero Osorio, M. R., Bouy, H., \& Wainscoat, R. 2006, ApJL, 644, L75

Mason, B. D., Wycoff, G. L., Hartkopf, W. I., Douglass, G. G., \& Worley, C. E. 2001, AJ, 122, 3466

Mermilliod, J.-C., \& Mermilliod, M. 1994, Catalogue of Mean UBV Data on Stars (Berlin: Springer-Verlag)

Monet, D. G., Levine, S. E., Canzian, B., et al. 2003, AJ, 125, 984 (USNO-B1.0)

Monnier, J. D., Che, X., Zhao, M., et al. 2012, ApJL, 761, L3

Morell, O. 1994, PhD thesis, Acta Universitas Upsaliensis

Murgas, F., Jenkins, J. S., Rojo, P., Jones, H. R. A., \& Pinfield, D. J. 2013, A\&A, 552, A 27

Neuhaeuser, R., Sterzik, M. F., Schmitt, J. H. M. M., et al. 1995, A\&A, 297, 391

Nordström, B., Mayor, M., Andersen, J., et al. 2004, A\&A, 418, 989

Parthasarathy, M., \& Lambert, D. L. 1987, JApA, 8, 51

Pecaut, M. J., \& Mamajek, E. E. 2013, ApJS, 208, 9

Pojmanski, G. 1997, AcA, 47, 467

Pollacco, D. L., Skillen, I., Collier Cameron, A., et al. 2006, PASP, 118, 1407

Press, W. H., Teukolsky, S. A., Vetterling, W. T., \& Flannery, B. P. 1992, Numerical Recipes in C: The Art of Scientific Computing (2nd ed.; New York: Cambridge Univ. Press)

Quillen, A. C. 2006, MNRAS, 372, L14

Rajpurohit, A. S., Reylé, C., Allard, F., et al. 2013, A\&A, 556, A15

Reid, I. N., Cruz, K. L., Allen, P., et al. 2003, AJ, 126, 3007

Reid, I. N., Gizis, J. E., \& Hawley, S. L. 2002, AJ, 124, 2721

Ribas, I. 2003, A\&A, 400, 297

Roeser, S., Demleitner, M., \& Schilbach, E. 2010, AJ, 139, 2440 (PPMXL)

Röser, S., Schilbach, E., Schwan, H., et al. 2008, A\&A, 488, 401 (PPMX)

Salim, S., \& Gould, A. 2003, ApJ, 582, 1011

Santos, N. C., Israelian, G., \& Mayor, M. 2004, A\&A, 415, 1153

Schlaufman, K. C., \& Laughlin, G. 2010, A\&A, 519, A105

Scholz, R.-D., Meusinger, H., \& Jahreiß, H. 2005, A\&A, 442, 211

Schönrich, R., Binney, J., \& Dehnen, W. 2010, MNRAS, 403, 1829

See, T. J. J. 1898, AJ, 18, 181

Shaya, E. J., \& Olling, R. P. 2011, ApJS, 192, 2

Skrutskie, M. F., Cutri, R. M., Stiening, R., et al. 2006, AJ, 131, 1163

Soderblom, D. R. 2010, ARA\&A, 48, 581

Soderblom, D. R., Nelan, E., Benedict, G. F., et al. 2005, AJ, 129, 1616

Stauffer, J. R., Hartmann, L. W., Fazio, G. G., et al. 2007, ApJS, 172, 663

Stauffer, J. R., Schultz, G., \& Kirkpatrick, J. D. 1998, ApJL, 499, L199

Tinney, C. G. 1996, MNRAS, 281, 644

Tokovinin, A. A. 1998, AstL, 24, 178

Torres, G., \& Ribas, I. 2002, ApJ, 567, 1140

Townsend, R. H. D., Owocki, S. P., \& Howarth, I. D. 2004, MNRAS, 350, 189 van Leeuwen, F. ed. 2007, Hipparcos, the New Reduction of the Raw Data (Astrophysics and Space Science Library, Vol. 350; Berlin: Springer)

Ventura, P., Zeppieri, A., Mazzitelli, I., \& D’Antona, F. 1998, A\&A, 334, 953

Voges, W., Aschenbach, B., Boller, T., et al. 1999, A\&A, 349, 389

Winters, J. G., Henry, T. J., Jao, W.-C., et al. 2011, AJ, 141, 21

Wright, E. L., Eisenhardt, P. R. M., Mainzer, A. K., et al. 2010, AJ, 140, 1868

Wroblewski, H., \& Costa, E. 1999, A\&AS, 139, 25

Yoon, J., Peterson, D. M., Kurucz, R. L., \& Zagarello, R. J. 2010, ApJ, 708, 71

Zacharias, N., Finch, C. T., Girard, T. M., et al. 2013, AJ, 145, 44 (UCAC4) 